\title{
Use of glyceraldehyde 3-phosphate dehydrogenase (GAPDH) as internal control in Western-blot experiments?
}

\begin{abstract}
Abbreviations: GAPDH, glyceraldehyde 3-phosphate
\end{abstract} dehydrogenase; NO, nitric oxide; ER, endoplasmic reticulum

\section{Letter to Editor}

For many years glyceraldehyde 3-phosphate dehydrogenase (GAPDH) was identified as a simple glycolytic enzyme. Particularly, GAPDH catalyzes the coinciding phosphorylation and oxidation of glyceraldehyde-3-phosphate to 1,3-biphosphoglycerate. ${ }^{1}$ Loading controls, often called "housekeeping genes", are known to be structural and definitely expressed at high levels in most of tissues and cells. They are used to normalize the level of protein detected by confirm that protein loading is the same across the gel. Discovering and verification of many proteins is often done using Western blotting by normalizing against "housekeeping proteins", such as GAPDH, $\beta$-actin, or $\beta$-tubulin, to emend for protein loading and factors, such as transfer efficiency. ${ }^{2}$ Recent studies, show that, besides its function in altering glyceraldehyde3-phosphate to 1, 3-biphosphoglycerate during glycolysis, GAPDH is involved in several non-metabolic processes, including transcriptional activation and cell apoptosis. ${ }^{3}$ Activated Akt2 may increase ovarian cancer cell survival via prevention of GAPDH-induced apoptosis. This effect of Akt 2 is partially mediated by its phosphorylation of GAPDH at Thr-237, which results in the prevention of GAPDH nuclear translocation. ${ }^{3}$ This study prove that GAPDH is S-nitrosylated by Nitric Oxide (NO) in response to cell stress, which result in, it to bind the protein Siah1, anubiquitin ligase. The complex moves into the nucleus where Siah1 targets nuclear proteins for degradation, thus beginning controlled cell shutdown. ${ }^{4}$

GAPDH also appears to be Participate in the vesicle transport from the endoplasmic reticulum (ER) to the Golgi apparatus, which is part of shipping route for secreted proteins. It was found that, GAPDH is recruited by rab2 to the vesicular-tubular clusters of the ER where it helps to form COP 1 vesicles. GAPDH is activated via tyrosinephosphorylation by Src. ${ }^{5}$ Thus, researchers should carefully assess the appropriate use of housekeeping gene-encoded proteins as internal standards to normalize samples for comparisons of different cell populations from subjects of different ages. ${ }^{6}$

Some investigations determined comparative proteins expression between MCF7 and SKBR3 that are epithelial breast cancer cell lines derived from pleural effusions, of two Caucasian women. ${ }^{7,8}$ We have previously identified a restricted number of differentially expressed proteins with an immunogenic property between SKBR3 and MCF7 cells using 2-DE and mass spectrometry including glyceraldehyde 3-phosphate dehydrogenase (GAPDH). ${ }^{78}$ We found that, the protein expression level of GAPDH is higher in SKBR3 cell line than MCF7 cell line. Therefore, to confirm these findings we used specific antibody against GAPDH in western-blot experiment (Figure 1). The results of this experiment confirm previous findings that GAPDH expression level is higher in SKBR3 cell line. In conclusion of this study we suggest that in utilization of GAPDH as internal control in western-blot experiments we must consider a large number of factors
Volume 2 Issue 6 - 2015

Ghasem Ghalamfarsa,' Faraz Zare, ${ }^{2}$ Zahra
Mojtahedi
'Cellular \& Molecular Research Center, Yasuj University of
Medical Sciences, Iran
${ }^{2}$ Institute for Cancer Research, Shiraz University of Medical
Sciences, Iran

Correspondence: Ghasem Ghalamfarsa, Cellular \& Molecular Research Center, Yasuj University of Medical Sciences, Mashhad University of Medical Science, Vakil-abad Blvd, Yasuj, Iran,Tel +989177416921,Email ghasem_ghalamfarsa@yahoo.com

Received: June 10,2015 | Published: July 09, 2015

such as pathological states, different tissues, different cell lines, cell cycle phase, differentiation or proliferation status, age, sex and stress conditions.

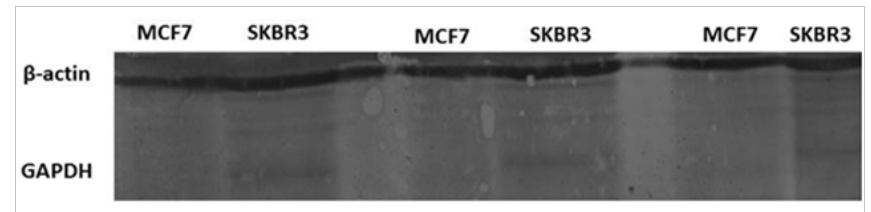

Figure I Comparative Immuno-blotting between two cell lines (MCF7 and SKBR3) of breast cancer. Cells were lysed in appropriate buffers and analyzed by Western blotting using specific mAb for glyceraldehyde-3-phosphate dehydrogenase (GAPDH) and $\beta$-actin.

\section{Acknowledgments}

None.

\section{Conflicts of interest}

The authors have no financial conflicts of interest to declare.

\section{Funding}

None.

\section{References}

1. Colell A, Green DR, Ricci JE. Novel roles for GAPDH in cell death and carcinogenesis. Cell Death Differ. 2009;16(12):1573-1581.

2. Ferguson RE, Carroll HP, Harris A, et al. Housekeeping proteins: A preliminary study illustrating some limitations as useful references in protein expression studies. Proteomics. 2005;5(2):566-571.

3. Huang Q, Lan F, Zheng Z, et al. Akt2 Kinase Suppresses Glyceraldehyde-3-phosphate Dehydrogenase (GAPDH)-mediated Apoptosis in Ovarian Cancer Cells via Phosphorylating GAPDH at Threonine 237 and Decreasing Its Nuclear Translocation. J Biol Chem. 2011;286(49):42211-42220. 
4. Hara MR, Agrawal N, Kim SF, et al. S-nitrosylated GAPDH initiates apoptotic cell death by nuclear translocation following Siah1 binding. Nat Cell Biol. 2005;7(7):665-674.

5. Tisdale EJ, Artalejo CR. A GAPDH mutant defective in Src-dependent tyrosine phosphorylation impedes Rab2-mediated events. Traffic. 2007;8(6):733-741.

6. Yu HR, Kuo HC, Huang HC, et al. Glyceraldehyde-3-phosphate dehydrogenase is a reliable internal control in Western blot analysis of leukocyte subpopulations from children. Anal Biochem. 2011;413(1):2429.
7. Zahra Mojtahedi NEaAG. Comparative Proteomics Analysis of SKBR3 and MCF7 Breast Cancer Cell Lines Using Two Dimensional Electrophoresis:Ready to Build Postgenomics Capacity for OMICS R\&D in Developing Countries? Current Pharmacogenomics and Personalized Medicine. 2012;10:132-137.

8. Mojtahedi Z, Safaei A, Yousefi Z, et al. Immunoproteomics of HER2Positive and HER2-Negative Breast Cancer Patients with Positive Lymph Nodes. OMICS. 2011;15(6):409-418. 